\title{
Routh-type Table Test for Zero Distribution of Polynomials with Commensurate Fractional and Integer Degrees
}

\author{
Shu Liang ${ }^{1}$, Sheng-Guo Wang ${ }^{2 *}$, and Yong Wang ${ }^{3}$
}

\begin{abstract}
This paper mainly presents Routh-type table test methods for zero distribution of polynomials with commensurate fractional degrees on the left-half plane, right-half plane and imaginary axis in the complex plane. The proposed tabular methods are derived for extension and generalization of the Routh test, which is widely used in controls for zero distribution of polynomials with integer degrees. Singular cases are discussed and handled efficiently and simply. Necessary and sufficient conditions for the second singular case are completely analyzed in terms of symmetric zeros. A particular property is revealed that a polynomial with commensurate fractional degrees without pure imaginary zero may still be stable in the presence of the second singular case, which is impossible for a real polynomial with integer degrees. Furthermore, we present a test to solve the zero distribution problem with respect to general sector region for polynomials with commensurate fractional degrees and real/complex coefficients. Finally, numerical examples are given to illustrate the correctness and effectiveness of the results. The proposed methods have broad application areas, including various systems, circuits and
\end{abstract}

This work was supported in part by NSF Grant 1115564 to Prof. Sheng-Guo Wang.

1 Shu Liang has been visiting at UNC Charlotte, Charlotte, NC 28223-0001, USA, since September 2013 as a co-educated PhD student supported by the China Scholarship Council and in part by NCDOT Grant RP2013-13, and is now with Key Laboratory of Systems and Control, Institute of Systems Science, Chinese Academy of Sciences, Beijing, 100190, China. sliang@amss.ac.cn.

2 Sheng-Guo Wang is with the COE and CCI, UNC Charlotte, NC 28223-0001, USA; and he is currently with CEE, Hong Kong Polytechnic University (PolyU), as a US Fulbright-PolyU Senior Scholar of 2016 - 2017 selected by The US Fulbright Foreign Scholar Board. swang@uncc.edu.

3 Yong Wang is with Dept. Automation, USTC, Hefei, Anhui, China. yongwang@ustc.edu.cn.

* Corresponding Author: Prof. Sheng-Guo Wang, swang@uncc.edu, Tel: 1-704-687-5063.

Submitted on 14-09-2015, Revised on 12-04-2016. 


\section{control.}

Keywords: zeros, polynomials, stability, fractional order systems, circuits, systems.

\section{INTRODUCTION}

Determining zero distribution of a real or complex polynomial is a mathematical problem with a long history and various solutions, and is very important for stability and performance analysis in control and systems areas. Among that, the celebrated Routh table test [1] (also see [2], pp. 177-185) is the most efficient method for determining the distribution of polynomial zeros in the left and right half complex planes as well as the imaginary axis. Furthermore, since Routh-type results/techniques have more implications and applications than the original polynomial zero distribution analysis, various research topics and results associated with the Routh test have been developed especially by control, circuit and system theorists. To mention a few, Ho, Datta and Bhattacharyya [3] generalized the Hermite-Biehler theorem to prove the Routh table and revealed a property to check whether a zero moves across the imaginary axis or not from the neighbor rows. That property was also obtained through a simple proof [4]. Lev-Ari, Bistritz and Kailath [5] proposed fast triangular factorization to evaluate the inertia of Bezoutian matrices with displacement structure, which leads to the Routh-Hurwitz and Schur-Cohn tests as well as more general test for regions with arbitrary circles and straight lines. Due to the extensive works, Routh test has been extended to solve zero distribution problems for complex polynomials (e.g., [5] and [6]) and subregions enclosed by various type of boundaries (e.g., which are transformed from imaginary axis by any rational function [7], or arbitrary lines, or segments [8]). Recently, Bistritz [9] presented fraction-free forms of the Routh test for complex and real integer polynomials

Technical development of Routh test also includes the treatment for singular cases. For the first singular case, the so-called epsilon-replacement method is widely known but requires careful 
treatment for the presence of some imaginary axis zeros [2], [10]. Some computationally efficient methods are proposed via left-shifting the corresponding row to eliminate those leading zero elements [8]. Furthermore, Pal and Kailath [11] provided a quite complete solution for all singular cases by analyzing the quasi-Hankel Bezoutions. Genin [12] presented a different and effective test by using the quotient polynomials obtained from the Euclid division, whereby the first singular case was incorporated into the regular non-singular cases. Recently, Choghadi and Talebi [13] proposed an additional check on the distance of the consecutive zero rows for the multiplicities of pure imaginary zeros when the second singular case occurred.

It is noticed that in addition to stability test, the normal Routh test also has wide applications such as to $H_{2} / H_{\infty}$ norm computation and model reduction [14], analysis of linear analog circuits [15], and many industrial examples [16].

Since the past decade, fractional order systems have attracted increasing attention from the mathematics, control, circuit and industrial community due to its physical existence and potential improvement to systems [17]-[26]. For instance, real capacitors (with different kinds of dielectrics) and coils (with inherent eddy current and hysteresis losses) are experimentally verified to have response characteristics of fractional orders [21], [22]. Recently, the existence of solutions to impulsive nonlinear fractional order systems and impulsive fractional differential inclusions has been investigated in [25] and [26], respectively.

One of the most fundamental problems in the field of fractional order systems is the stability analysis. Matignon theorem [27] and its generalization [28] indicate that a linear time invariant fractional order system is BIBO stable if and only if its characteristic function as a polynomial with fractional degrees (PFD) has no closed-RHP zero. Thereafter, the stability and stabilization of fractional order systems have been extensively investigated, e.g., by LMI-based methods [29], 
[30], root locus method [31], analysis for rational order systems [32], and a review on various systems including linear or nonlinear, distributed, and time delay [33]. The classical Routh-Hurwitz criterion has been directly utilized for checking the stability of fractional order systems with commensurate order less than one [34], whereas that test is only sufficient and therefore conservative. A stability criterion in terms of some coefficient inequalities has been presented in [35], which is only applicable to a polynomial with commensurate fractional degrees (PCFD) with no more than three terms. Recently, Sabatier, Farges and Trigeassou [36] proposed a graph-based test that utilized a sequence of Nyquist plots, whereby the stability of non-commensurate fractional order systems were able to be checked.

Despite these remarkable achievements for stability of fractional order systems, none of them investigates a test from tabular procedures yielding necessary and sufficient stability and/or zero distribution criterion for general PCFDs as we present in this paper.

It is noticed that existing zero solving algorithms implemented in modern computers (e.g., Matlab command "roots" based on the QR iteration algorithm) are powerful to obtain the zero location of certain polynomials. However, these numerical methods with iteration process may suffer unstable or accuracy problem for high order polynomials (especially ill-conditioned polynomials with multiple zeros) as shown in our example 3 and pointed out by [37] as "In these cases, the solution causes problems for the available software, and this motivates further research on the design of effective algorithms for solving zeros of polynomial". Furthermore, it is well known that it is impossible to have a closed form of algebraic expression for the zeros of general polynomials with degree higher than four. Therefore, any zero solving method and algorithm for general polynomials are some approximation ways in theoretical and essential sense, i.e., not exact, in addition to their numerical calculation approximation. On the other hand, the Routh-type test 
can be used as a necessary and sufficient condition for stability with $O\left(n^{2}\right)$ arithmetic calculations. More important is that its method and algorithm are accurate and efficient in both theoretical and essential sense. It is noticed that another widely used stability test via LMI (e.g., the Lyapunov inequality) without zero solving has $O\left(n^{3}\right)$ computational complexity in one search iteration with a predefined accuracy to determine and stop the iteration process [38].

The Routh test is powerful for obtaining zero distribution of polynomials with integer degrees by arithmetic operations of the coefficients without actually solving zeros, having extremely low computational complexity and revealing the analytical relationship between the coefficients and zero distribution for dynamic behaviors, which can be applied for system design problems. As fractional order systems are considered, a natural question is: what is a Routh-type table test for the PCFDs? Technically, how to treat the various possible singular cases for the PCFDs and to provide a complete test? How do the singular cases imply zeros and/or their distributions?

This paper is devoted to addressing the above challenging questions and problems, and presenting the solutions. We derive a new Routh-type table test for polynomials with commensurate fractional degrees, including the complete treatment to all singular cases. Moreover, we consider general sector regions and PCFDs with either real or complex coefficients. The reason to consider complex polynomials is that they arise when we consider zeros of system characteristic function in specific sectors for guaranteeing specific performance, e.g., damping ratios. The proposed Routh-type test has further advantages, e.g., to system parameter design. Therefore it makes the approach stronger over zero solving methods and graph-based methods. Moreover, it provides more accurate result with less computation time than other methods.

The main contributions of this paper include the followings: (1) deriving a Routh-type table test for zero distribution of polynomials with commensurate fractional degrees, which is also 
complete for all possible singular cases; (2) revealing the symmetric zeros distribution of PCFDs when the second singular case occurs; (3) extending the proposed test to general sector regions and for complex PCFDs; and (4) demonstrating their applications to circuits and systems by examples.

The authors acknowledge the viewpoint, i.e., usually it seems not easy to offer a new contribution to an old topic like Routh test with more than 100 years history and enormous amount of literature. Even though no literature considers any PCFD via a Routh-type stability test, a PCFD can be transformed into a polynomial of integer degrees together with the corresponding zero distribution region as some sector (not the left half plane) for its stability test. A Routh test of integer degree polynomials for the sector region is available at least from the work by Sherman (1946) [39], also Marden's monograph (1966) [40] and some late developments such as by Tsai and Chen [8]. In contrast, since the characteristic PCFD determines the stability and dominates the performance of a fractional order system, we directly consider the PCFD to investigate how classical Routh test approach/technique can be generalized to PCFDs with its theoretic proof. It is revealed by our work that the zero distribution of a PCFD with respect to any sector region in the Riemann surface can be completely solved by tabular method as a Routh-type test, whereas the transformation approach via available integer degree Routh table would encounter difficulties for counting zeros when the mapping $s^{\alpha} \rightarrow \lambda$ is not one-to-one. Thus, we will not discuss an approach via the current Routh table for PCFDs here, but in a future separate paper. Therefore, our goal is to provide a new contribution with a new approach.

Here, we discuss and develop our new methods to address these issues directly via our uniform Routh-type table formula valid for both integer and commensurate fractional degrees polynomials. The rest of this paper is organized as follows. Section 2 gives preliminaries for PFDs and their corresponding zeros. In Section 3, the main test and an auxiliary test are presented for 
zero distribution of real PCFD with respect to the RHP, LHP and imaginary axis of the Riemann principal sheet. In Section 4, the singular cases are analyzed completely. In Section 5, a general test is provided for the zero distribution of real or complex PCFDs with respect to general sector regions. In Section 6, examples are given to illustrate the efficient features of the proposed tests for various types of polynomials and cases. Finally, Section 7 concludes this paper. The main theoretical proofs are in an Appendix.

Notations: $\mathbb{N}_{\text {ood }}, \mathbb{N}, \mathbb{Z}, \mathbb{R}, \mathbb{R}^{+}$and $\mathbb{C}$ stand for odd, natural, integer, real, positive real and complex number sets, respectively. Notation ged stands for greatest common divisor. The symbol " $"$ is subtraction operator for sets, and “*” is conjugate symbol for complex number. Symbol $\operatorname{deg}(f)$ stands for the highest degree of a normal polynomial or a PFD $f$. Cauchy index of a real rational function $H(x)$ between the limits $a$ and $b$ is denoted by $I_{a}^{b} H(x)$.

\section{PRELIMINARIES}

In this section, we give a general description for polynomials of fractional degrees (PFDs) and their zeros. A PFD can be written as

$$
Q(s)=q_{m} s^{\beta_{m}}+q_{m-1} s^{\beta_{m-1}}+\cdots+q_{1} s^{\beta_{1}}+q_{0}, q_{m} \neq 0
$$

where $0<\beta_{1}<\cdots<\beta_{m} \in \mathbb{R}^{+}$, and $q_{0}, q_{1}, \cdots, q_{m} \in \mathbb{C}$. If $q_{0}=0$, we write $Q(s)=s^{\beta_{1}} Q_{1}(s)$ for some $\beta_{1} \in \mathbb{R}^{+}$and $Q_{1}(s)$ with a nonzero constant item. Then $Q(s)$ has $\beta_{1}$ zeros at origin if $\beta_{1} \in \mathbb{N}$, or $\beta_{1}$-multiple essential singularity at origin if $\beta_{1} \in \mathbb{R}^{+} \backslash \mathbb{N}$. After this treatment, we may assume that the considered PFD has a nonzero constant item.

The $Q(s)$ in (1) is said to be commensurate if there exists $\alpha \in \mathbb{R}^{+}$such that $\beta_{1} /$ $\alpha, \cdots, \beta_{m} / \alpha \in \mathbb{N}$. Thus a commensurate PFD (PCFD) with $\beta_{m}=n \alpha$ can be written as

$$
P(s)=p_{n} s^{n \alpha}+p_{n-1} s^{(n-1) \alpha}+\cdots+p_{1} s^{\alpha}+p_{0}, p_{0} \neq 0
$$

where $p_{0}, p_{1}, \cdots, p_{n} \in \mathbb{C}$. 
To investigate the zeros structure of $P(s)$, one may employ its companion polynomial $P_{c}(\cdot)$ from transformation $\lambda=s^{\alpha}$ as

$$
P_{c}(\lambda) \triangleq p_{n} \lambda^{n}+p_{n-1} \lambda^{n-1}+\cdots+p_{1} \lambda+p_{0}, p_{0} \neq 0
$$

The $P_{c}(\lambda)$ is a polynomial of degree $n$ and has $n$ zeros as $\lambda_{l}=r_{l} e^{j \theta_{l}}, r_{l}>0, l=1,2, \cdots, n$, in view of $p_{0} \neq 0$. Thus $P(s)$ has zeros

$$
s=\lambda_{l}^{1 / \alpha}=r_{l}^{1 / \alpha} e^{j\left(\theta_{l}+2 k \pi\right) / \alpha}, k \in \mathbb{Z}, l=1,2, \cdots, n .
$$

For $\alpha=1$, integer degree $P(s)$ is an analytical function and has exactly $n$ zeros $\lambda_{l}$, $l=1,2, \cdots, n$, in the complex plane. For $\alpha \notin \mathbb{N}, P(s)$ becomes a multivalued function due to $\ln (s)$, i.e., $s^{\alpha}=e^{\alpha \cdot \ln (s)}$, and has infinite number of zeros as shown in (4). To analyze both the global and local behaviors of PFD, the following structural Riemann surface is introduced.

Definition 1: The Riemann surface $\mathcal{R S}$ is $\mathcal{R} \mathcal{S} \triangleq\{s:-\infty<\arg \{s\}<+\infty\}=\bigcup_{k \in \mathbb{Z}} \mathcal{R} \mathcal{S}_{k}$, where the $k$ th Riemann sheet $\mathcal{R} \mathcal{S}_{k}$ is defined as $\mathcal{R} \mathcal{S}_{k} \triangleq\{s:-\pi+2 k \pi<\arg \{s\} \leq \pi+2 k \pi\}$ for any $k \in \mathbb{Z}$. Particularly, the Riemann principal sheet $\mathcal{R P} \mathcal{S}$ is defined as the central Riemann sheet as $\mathcal{R P} \mathcal{S} \triangleq \mathcal{R} \mathcal{S}_{0}=\{s:-\pi<\arg \{s\} \leq \pi\}$, i.e., $k=0$.

A commensurate or non-commensurate PFD has its single-valued and analytic branch for $s$ belonging to each $\mathcal{R} \mathcal{S}_{k}$. Among them, the branch in $\mathcal{R} \mathcal{P} \mathcal{S}$ is utilized to describe fractional order systems, because it determines the Cauchy principal value of the integral corresponding to the inverse Laplace transformation of system transfer function. The direct application of residue theorem implies that only the zeros in $\mathcal{R P} \mathcal{S}$ of the PFD determine the system stability and dynamic performance. Therefore, we particularly focus on RHP of $\mathcal{R P} \mathcal{S}$ and present the corresponding test first. Our general test is applicable for any sector region of the $\mathcal{R P} \mathcal{S}$ as well as any sector region of the entire $\mathcal{R} \mathcal{S}$.

Definition 2: Among the zeros of a PFD, those in $\mathcal{R P} \mathcal{S}$ are called principal zeros (also 
called structural zeros in [27]). We further call adjoint zeros for those zeros not in $\mathcal{R} \mathcal{P} \mathcal{S}$.

Definition 3: A triple $\left(n_{l}, n_{i}, n_{r}\right)$ is called the zero distribution triple of a given PFD $f$, where $n_{l}(f), n_{i}(f)$, and $n_{r}(f)$ stand for the numbers of principal zeros on the LHP, imaginary axis and RHP, respectively. Symbol $n_{p}(f)$ stands for the total number of principal zeros, i.e., $n_{p} \triangleq n_{l}+n_{i}+n_{r}$

Lemma 1 ([27], [28]): For a fractional order system with its fractional degree characteristic polynomial function $P(s)$ in (2), that is stable if and only if $0<\alpha<2$ and all zeros $\lambda_{l}(l=1,2, \cdots, n)$ of the companion polynomial $P_{c}(\lambda)$ in (3) satisfy the condition $\left|\arg \left(\lambda_{l}\right)\right|>$ $\alpha \pi / 2$

An immediate consequence of Lemma 1 and equation (4) leads to an essential stability criterion of a fractional order system with its characteristic function $P(s)$, that is if and only if $n_{i}(P(s))=0$ and $n_{r}(P(s))=0$, i.e., no its principal zero in the closed right half plane.

Remark 1: The system characteristic function $P(s)$ in (2) has a clearer direct physical meaning than $P_{c}(\lambda)$ in (3), and $P(s)$ should play an important role in system analysis. It is because $P(s)$ is from the Laplace transform, where the Laplace variable $s$ directly reflects/contains the time response decay and frequency information by its real part and imaginary part respectively. This observation is one of our motivations to develop analytical approach (via a Routh-type test) for $P(s)$. In addition, our goal is to treat more generalized zero distribution problem with respect to any degree PCFDs, including the limited degree case with $0<\alpha<2$ as well. Furthermore, the presenting Routh-type tests will reveal the relationship between those polynomial coefficients and their zeros distribution, that is useful for system design as shown by Example 2 in Section 6, and that the zero solving method may not provide. 


\section{ROUTH-TYPE TESTS FOR REAL COEFFICIENT AND COMMENSURATE PFDS}

In this section, we focus on zero distribution test with respect to RHP, imaginary axis and LHP of $\mathcal{R P S}$ for $P(s)$ in (2) with real coefficients.

\section{Main Test}

Step 1:

If $n \alpha \in \mathbb{R}^{+} \backslash \mathbb{N}_{\text {odd }}$, set a parameter $\rho=1$ [to indicate the case of $\operatorname{deg}(R(\omega)) \geq \operatorname{deg}(I(\omega))$ ], and

$$
a_{k}=p_{k} \cos \left(\frac{k \pi \alpha}{2}\right), b_{k}=p_{k} \sin \left(\frac{k \pi \alpha}{2}\right), k=0,1, \cdots, n
$$

Else ( $n \alpha \in \mathbb{N}_{\text {odd }}$ ), set $\rho=0$ and

$$
a_{k}=p_{k} \sin \left(\frac{k \pi \alpha}{2}\right), b_{k}=p_{k} \cos \left(\frac{k \pi \alpha}{2}\right), k=0,1, \cdots, n
$$

Then, firstly write a table as

$$
\begin{array}{lllll}
a_{n} & a_{n-1} & \cdots & a_{1} & a_{0} \\
b_{n} & b_{n-1} & \cdots & b_{1} & b_{0}
\end{array} .
$$

If all $b_{k}$ are zeros, i.e., an entire zero row, go to Step 3.

Else if the first element in a row is zero, then do an operation O: "left shift this row until the first element of this row is not zero". For example, suppose the current row is " $0,0,1,4,3$ ", then operation O yields " $1,4,3,0,0$ ". Then go to Step 2.

Step 2:

Calculate a next row $\mathbf{c}$ using the current last two rows of $\mathbf{a}$ and $\mathbf{b}$ as follows.

$$
\begin{array}{lllll}
a_{n} & a_{n-1} & \cdots & a_{1} & a_{0} \\
b_{n} & b_{n-1} & \cdots & b_{1} & b_{0}, \\
c_{n} & c_{n-1} & \cdots & c_{1} & c_{0}
\end{array}
$$

where 


$$
c_{k}=\frac{a_{n} b_{k-1}-b_{n} a_{k-1}}{b_{n}}, k=1,2, \cdots, n \text {, and } c_{0}=0 .
$$

The calculation in (9) is different from the classical Routh table algorithm by a negative sign.

If row $\mathbf{c}$ is an entire zero row, go to Step 3.

Else, do operation $\mathrm{O}$ if $c_{n}=0$, and go back to Step 2 .

Step 3:

Mark the row immediately above the current entire zero row (with $\diamond$ ) as

$$
\begin{array}{ccccc}
\vdots & \vdots & \cdots & \vdots & \vdots \\
\diamond h_{n} & h_{n-1} & \cdots & h_{1} & h_{0} . \\
0 & 0 & \cdots & 0 & 0
\end{array}
$$

Then go to Step 4. (This symbol $\diamond$ marks on the row immediately above the zero row at the first time only for late calculation in Step 6 when the second singular case occurs.)

\section{Step 4:}

Represent the row immediately above this entire zero row by renumbering their subscripts in the following, where $e_{0} \neq 0$ is the first nonzero tail entry in this row,

$$
\begin{array}{cccccccc}
e_{q} & e_{q-1} & \cdots & e_{1} & e_{0} & 0 & \cdots & 0 \\
0 & 0 & \cdots & 0 & 0 & 0 & \cdots & 0
\end{array} .
$$

If $q=0$, then row $\mathbf{e}$ is the end row of the entire table and go to Step 6 .

Else, replace the current entire zero row from (11) as

$$
\begin{array}{cccccccc}
e_{q} & e_{q-1} & \cdots & e_{1} & e_{0} & 0 & \cdots & 0 \\
q e_{q} & (q-1) e_{q-1} & \cdots & e_{1} & 0 & 0 & \cdots & 0
\end{array} \text {. }
$$

Then go to Step 5.

Step 5:

Calculate next row $\mathbf{c}$ using the current last two rows $\mathbf{a}$ and $\mathbf{b}$ as same as Step 2.

If row $\mathbf{c}$ is an entire zero row, go to Step 4.

Else, do operation O if necessary, and go back to Step 5. 
Step 6:

The final table (13) is given at the top of this page,

$\begin{array}{cccccccccc} & x_{1, n} & x_{1, n-1} & \cdots & \cdots & \cdots & x_{1, m_{1}} & \cdots & 0 & \\ & x_{2, n} & x_{2, n-1} & \cdots & \ldots & x_{2, m_{2}} & \cdots & \cdots & 0 & \\ V_{f} & \vdots & \vdots & \cdots & \vdots & \vdots & \ddots & \cdots & \vdots & V_{l} \\ & \diamond x_{g, n} & x_{g, n-1} & \cdots & x_{g, m_{g}} & 0 & \cdots & \cdots & 0 & \\ & x_{g+1, n} & \cdots & x_{g+1, m_{g+1}} & 0 & \cdots & \cdots & \cdots & 0 & \\ V_{d f} & \vdots & \therefore & \therefore & \ddots & \vdots & \vdots & \vdots & \vdots & V_{d l} \\ & x_{h, m_{h}} & 0 & \cdots & \cdots & \cdots & \cdots & \cdots & 0 & \end{array}$

where $x_{1, m_{1}} \cdots x_{h, m_{h}}$ are the first nonzero tail element in each row. Numbers $V_{f}, V_{l}, V_{d f}$ and $V_{d l}$ are the sign change indices as defined as follows.

$$
\begin{gathered}
V_{f} \triangleq V\left(x_{1, n}, x_{2, n}, \cdots, x_{g, n}\right), \\
V_{l} \triangleq V\left(x_{1, m_{1}}, x_{2, m_{2}}, \cdots, x_{g, m_{g}}\right), \\
V_{d f} \triangleq V\left(x_{g, n}, x_{g+1, n}, \cdots, x_{h, m_{h}}\right), \\
V_{d l} \triangleq V\left(x_{g, m_{g}}, x_{g+1, m_{g+1}}, \cdots, x_{h, m_{h}}\right),
\end{gathered}
$$

where $V(a, b, \ldots, e)$ is the number of sign changes in the sequence $(a, b, \ldots, e)$. Set

$$
\sigma \triangleq \operatorname{sgn}\left(x_{1, m_{1}} \cdot x_{2, m_{2}}\right)
$$

where $\operatorname{sgn}(\cdot)$ is the sign function. And set

$$
d \triangleq V_{d l}-V_{d f}
$$

Finally, we get these $d, \sigma, \rho, V_{f}$ and $V_{l}$. This is the end of the Main test.

Theorem 1: For $P(s)$ in (2) with real coefficients, its pure imaginary zeros number $n_{i}$ and RHP zeros number $n_{r}$ are as follows.

$$
n_{i}(P)=2 d=2\left(V_{d l}-V_{d f}\right), \quad n_{r}(P)=\left\langle\frac{n \alpha}{2}-d-\frac{\sigma}{2}(1-\rho)-(2 \rho-1)\left(V_{f}-V_{l}\right)\right\rangle
$$




$$
=\left\{\begin{array}{l}
\left\langle\frac{n \alpha}{2}\right\rangle-d-\left(V_{f}-V_{l}\right), \quad \text { for } n \alpha \in \mathbb{R}^{+} \backslash \mathbb{N}_{\text {odd }} \\
\frac{n \alpha}{2}-\frac{\sigma}{2}-d+\left(V_{f}-V_{l}\right), \text { for } n \alpha \in \mathbb{N}_{\text {odd }}
\end{array},\right.
$$

where $d, \sigma, \rho, V_{f}$ and $V_{l}$ are obtained from the above Main test, and $\langle\cdot\rangle$ is a well-known round-off operator, e.g.,

$$
\langle 4.4\rangle=4, \text { and }\langle 4.5\rangle=5
$$

Proof: Please see Proof of Theorem 1 in the Appendix.

The Main Test can be regarded as a stability test since it counts the number of principal zeros in the closed-RHP. However, if the zero distribution is concerned, we also need the total number $n_{p}$ of principal zeros to calculate $n_{l}$. The following Auxiliary Test is given to solve this problem.

\section{Auxiliary Test}

If $2 n \alpha \in \mathbb{R}^{+} \backslash \mathbb{N}_{\text {odd }}$, set $\rho=1$ and

$$
a_{k}=p_{k} \cos (k \pi \alpha), b_{k}=p_{k} \sin (k \pi \alpha), k=0,1, \cdots, n
$$

Else, set $\rho=0$ and

$$
a_{k}=p_{k} \sin (k \pi \alpha), b_{k}=p_{k} \cos (k \pi \alpha), k=0,1, \cdots, n
$$

Then, firstly write a table as

$$
\begin{array}{lllll}
a_{n} & a_{n-1} & \cdots & a_{1} & a_{0} \\
b_{n} & b_{n-1} & \cdots & b_{1} & b_{0}
\end{array} .
$$

The remaining part is as same as the above Main Test to get its $\rho, \sigma, V_{f}$ and $V_{l}$. Here, Steps 3 to 5 can be skipped. It does not need to calculate the number $d$. This is the end of Auxiliary test.

Theorem 2: For $P(s)$ in (2) with real coefficients, its total principal zeros number $n_{p}$ is

$$
\begin{aligned}
& n_{p}=\left\langle n \alpha-\sigma(1-\rho) / 2-(2 \rho-1)\left(V_{f}-V_{l}\right)\right\rangle \\
& =\left\{\begin{array}{l}
\langle n \alpha\rangle-\left(V_{f}-V_{l}\right), \quad \text { for } 2 n \alpha \in \mathbb{R}^{+} \backslash \mathbb{N}_{\text {odd }} \\
n \alpha-\sigma / 2+\left(V_{f}-V_{l}\right), \text { for } 2 n \alpha \in \mathbb{N}_{\text {odd }}
\end{array}\right.
\end{aligned}
$$

where $\rho, \sigma, V_{f}$ and $V_{l}$ are obtained from Auxiliary test. 
Proof: Please see Proof of Theorem 2 in the Appendix.

Remark 2: From the Main Test and the Auxiliary Test, we have $n_{l}=n_{p}-n_{i}-n_{r}$, which completes the zero distribution triple $\left(n_{l}, n_{i}, n_{r}\right)$.

Remark 3: The computational complexity of our Main or Auxiliary Test is $n^{2}+O(n)$. It involves $2(n-1)$ sine/cosine calculations, (each one with relative error $O\left(2^{-q}\right)$ has $O(M(q) \log (q))$ operations, where $M(q)$ is the number of single-precision operations required to multiply q-bit integers), $n^{2}$ table elements calculations (the calculation in (9) for each element has two multiplications, one division and one subtraction), and about $2 n$ logical judgment times. Moreover, for integer degree polynomials, i.e., with commensurate $\alpha=1$, the even columns must be all zeros and needs not to be calculated. Thus our Routh-type table also has only $0.25 n^{2}+O(n)$ computational complexity as same as classical Routh test for integer degree polynomials.

\section{ANALYSIS OF SINGULAR CASES}

There may be two kinds of singular cases in our Routh-type table: (i) a zero element in the first column, and (ii) an entire zero row. It is seen that the proposed tests treat the first singular case simply by the shifting operation $\mathrm{O}$.

Now we give a complete analysis for the second singular case.

Theorem 3: Consider $P(s)$ in (2) with real coefficients. The second singular case occurs in the Main Test if and only if there exists some $\gamma \in \mathcal{R S}$ such that $P(s)=0$ for all $s \in\left\{j \gamma, j \gamma^{*}\right.$, $\left.-j \gamma,-j \gamma^{*}\right\}$. Moreover, all such existing $\gamma$ s are exactly the common zeros of real and imaginary polynomials of $P(j \omega)$, i.e., the zeros of their greatest common divisor.

Proof: Please see Proof of Theorem 3 in the Appendix.

Remark 4: If $P(s)$ is a real polynomial with integer degrees, then the necessary and 
sufficient condition for the second singular case in Theorem 3 reduces to the well-known existence of zeros on the imaginary axis or "four (or two) symmetric zeros cluster" about both real and imaginary axes. Then the best conclusion one can expect for the corresponding system is marginal stable, or unstable. On the other hand, if $P(s)$ is a real PCFD, these "four (or two) symmetric zeros" can be either all principal zeros, or all adjoint zeros, or combining principal and adjoint zeros. In other words, since the adjoint zeros do not affect stability, a PCFD $P(s)$ can still be stable in presence of the second singular case (please see Example 1).

It will be much clearer to present the necessary and sufficient condition for the second singular case of $P(s)$ in (2) in terms of symmetric zeros of its companion polynomial $P_{c}(\lambda)$ in (3) as follows.

Proposition 1: The second singular case occurs for $P(s)$ in (2) with real coefficients if and only if $P_{c}(\lambda)$ in (3) has zeros on or symmetric about $\{\lambda: \arg \{\lambda\}= \pm \alpha \pi / 2\}$ in Fig. 1.

Proof: It follows from Theorem 3 that the second singular case implies a pair of symmetric zeros $s_{1}$ and $s_{2}$ that $\left|s_{1}\right|=\left|s_{2}\right|$ and $\arg \left\{s_{1}\right\}+\arg \left\{s_{2}\right\}= \pm \pi$. Let $\lambda_{1}=s_{1}^{\alpha}$ and $\lambda_{2}=s_{2}^{\alpha}$, then $P_{c}\left(\lambda_{1}\right)=P_{c}\left(\lambda_{2}\right)=0$ and $\left(\arg \left\{\lambda_{1}\right\}+\arg \left\{\lambda_{2}\right\}\right) / 2= \pm \alpha \pi / 2$.

\section{ROUTH-TYPE TEST FOR SECTOR REGIONS}

In this section, we consider the zero distribution of $P(s)$ in (2) with respect to a general sector region

$$
\Omega\left(\theta_{1}, \theta_{2}\right) \triangleq\left\{s: \theta_{1}<\arg \{s\}<\theta_{2}\right\} \subset \mathcal{R} S
$$

as shown in Fig. 2. Notation $n_{\Omega}$ stands for the number of zeros in $\Omega$, and $n_{\partial \Omega}^{(1)}$ and $n_{\partial \Omega}^{(2)}$ stand for the numbers of zeros on the edges $\left\{s: \arg \{s\}=\theta_{1}\right\}$ and $\left\{s: \arg \{s\}=\theta_{2}\right\}$, respectively. 


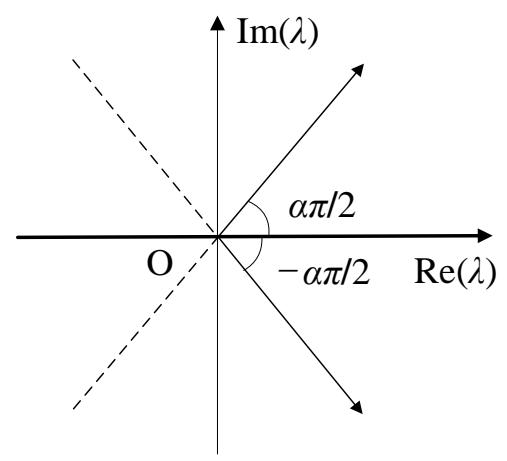

Fig. 1. Symmetric axes in $\lambda$-plane for the second singular case

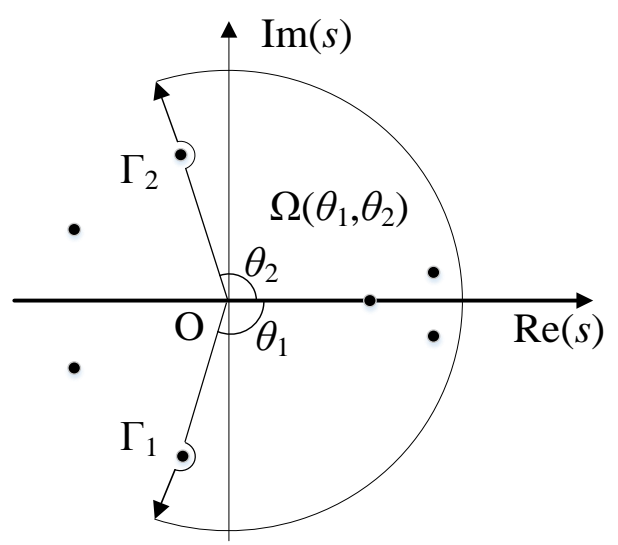

Fig. 2. General sector region $\Omega\left(\theta_{1}, \theta_{2}\right)$

\section{General Test}

Step 1:

Let $R(\omega) \triangleq \operatorname{Re}\left(P\left(e^{j \theta_{1}} \omega\right)\right)$ and $I(\omega) \triangleq \operatorname{Im}\left(P\left(e^{j \theta_{1}} \omega\right)\right)$, corresponding to the considered $\theta_{1}$. Then write a table as

$$
\begin{array}{lllll}
a_{n} & a_{n-1} & \cdots & a_{1} & a_{0} \\
b_{n} & b_{n-1} & \cdots & b_{1} & b_{0}
\end{array}
$$

where $\left\{a_{k}\right\}$ and $\left\{b_{k}\right\}$ are coefficients of $R(\omega)$ and $I(\omega)$ if $\operatorname{deg}(R(\omega)) \geq \operatorname{deg}(I(\omega))$, or the versa. Set $\rho_{1}=1$ if the former case, and set $\rho_{1}=0$ if the versa.

Next, do the same procedure as the Main Test until the numbers $d, \sigma, V_{f}$, and $V_{l}$ are obtained. And let $d_{1} \triangleq d, \Phi_{1} \triangleq V_{f}-V_{l}$, and $\sigma_{1} \triangleq \sigma$. 


\section{Step 2:}

For the corresponding $\theta_{2}$, do the same as Step 1, and get $\rho_{2}, d_{2}, \Phi_{2}$ and $\sigma_{2}$. This is the end of General Test.

Theorem 4: For zero distribution of $P(s)$ in (2) with respect to the sector region $\Omega\left(\theta_{1}, \theta_{2}\right)$ in (26), the numbers $n_{\partial \Omega}^{(1)}, n_{\partial \Omega}^{(2)}$ and $n_{\Omega}$ are as follows

$$
n_{\partial \Omega}^{(1)}=d_{1}, \quad n_{\partial \Omega}^{(2)}=d_{2}, \quad n_{\Omega}=\left\langle\Phi_{0}+\left(\rho_{1}-0.5\right) \Phi_{1}-\left(\rho_{2}-0.5\right) \Phi_{2}\right\rangle,
$$

where $\Phi_{0}=\frac{\left(\theta_{2}-\theta_{1}\right) n \alpha}{2 \pi}-\frac{d_{1}+d_{2}}{2}-\frac{\sigma_{2}\left(1-\rho_{2}\right)-\sigma_{1}\left(1-\rho_{1}\right)}{4}$, these $d_{1}, d_{2}, \rho_{1}, \rho_{2}, \Phi_{1}, \Phi_{2}, \sigma_{1}$ and $\sigma_{2}$ are obtained from the General test.

Proof: Please see Proof of Theorem 4 in the Appendix.

The second singular case in the General Test can occur if there are symmetric zeros with respect to the edge $\{s: \arg \{s\}=\theta\}, \theta=\theta_{1}$ or $\theta_{2}$. We have the following result with its proof omitted since it is similar to the proofs of Theorem 3 and Proposition 1.

Proposition 2: Consider the General Test for $P(s)$ in (2) with respect to sector region $\Omega\left(\theta_{1}, \theta_{2}\right)$ in (26). The following three statements are equivalent:

1. The second singular case occurs in a table with respect to $\theta=\theta_{1}$ or $\theta=\theta_{2}$;

2. There exists some $\gamma \in \mathcal{R} S$ such that $P(s)=0$ for both $s \in\left\{e^{j \theta} \gamma, e^{j \theta} \gamma^{*}\right\}$. Moreover, all these $\gamma \mathrm{s}$ are exactly the common zeros of the real and imaginary part functions of $P\left(e^{j \theta} \omega\right)$;

3. Its companion polynomial $P_{c}(\lambda)$ in (3) has zeros on or symmetric about $\{\lambda: \arg \{\lambda\}=\theta \alpha\}$.

Remark 5: In general, we may need to calculate two Routh-type tables to determine the zeros distribution of a PCFD with respect to a sector region in view of various different $\theta_{1}$ and $\theta_{2}$, as Theorem 4 states. However, from engineering viewpoint, above sector region should be considered with $\theta_{1}=-\theta_{2}$, i.e., a symmetric sector region $\Omega(-\theta, \theta)$ about the real axis for any real systems. Then, a symmetric table for either $\theta_{1}$ or $\theta_{2}$ does not need to be calculated if real 
PCFDs are considered. Moreover, when $\theta=\pi / 2$ (or $\pi$ ), the General Test further reduce to the Main (or Auxiliary) Test.

\section{EXAMPLES}

Example 1: This example is to illustrate our test and verify the relationship between the second singular case and symmetric zeros. Consider the following PCFD with commensurate $\alpha=0.5$ :

$$
P(s)=s^{2}+1.5714 s^{1.5}+1.2346 s+1.5714 s^{0.5}+1
$$

The Main Test has the table as:

$\begin{array}{ccccc}-1 & -1.1111 & 0 & 1.1111 & 1 \\ 1.1111 & 1.2346 & 1.1111 & 0 & 0 \\ \diamond-1 & -1.1111 & -1 & 0 & 0 \\ -2 & -1.1111 & 0 & 0 & 0 \\ 0.5556 & 1 & 0 & 0 & 0 \\ -2.4888 & 0 & 0 & 0 & 0 \\ -1 & 0 & 0 & 0 & 0\end{array}$

It yields $n_{i}=2 d=2\left(V_{d l}-V_{d f}\right)=0$, and $n_{r}=0$. To find $n_{l}$, we run the table of Auxilairy Test for $n_{p}$ first as:

$\begin{array}{ccccc}1 & 0 & -1.2346 & 0 & 1 \\ -1.5714 & 0 & 1.5714 & 0 & 0 \\ 0.2346 & 0 & -1 & 0 & 0 \\ 5.1268 & 0 & 0 & 0 & 0 \\ 1 & 0 & 0 & 0 & 0\end{array}$

It yields $n_{p}=2$. Finally, the principal zero distribution is $(2,0,0)$. In fact, all the principal zeros of $P(s)$ are $s_{1,2}=e^{ \pm j 7 \pi / 8}$. The mark $\diamond$ indicates that the second singular case occurs. To illustrate Theorem 3 as in Fig. 3, we observe a pair of adjoint zeros $s_{3,4}=e^{ \pm j 15 \pi / 8}$ of $P(s)$ such that $\left\{s_{1,2}, s_{3,4}\right\}=\left\{j \gamma, j \gamma^{*},-j \gamma,-j \gamma^{*}\right\}$ where $\gamma=e^{j 11 \pi / 8}$. In addition, to illustrate Proposition 1 as in Fig. 4, we observe that the companion polynomial $P_{c}(\lambda)$ has four zeros $\lambda_{1,2}=e^{ \pm j 7 \pi / 16}$ 
and $\lambda_{3,4}=e^{ \pm j 15 \pi / 16}$.

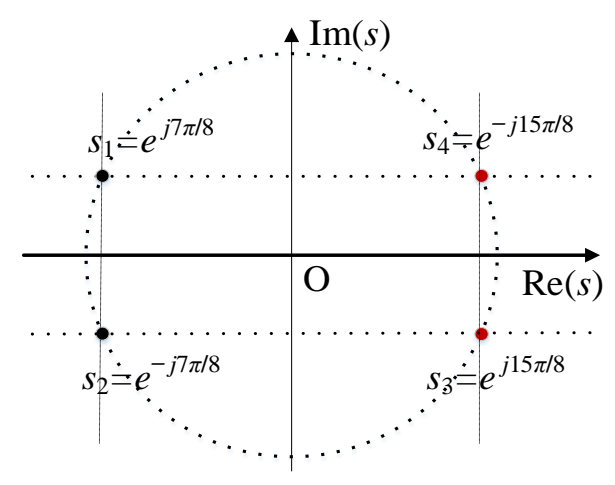

Fig. 3. Zero distribution of $P(s)$ in Example 1

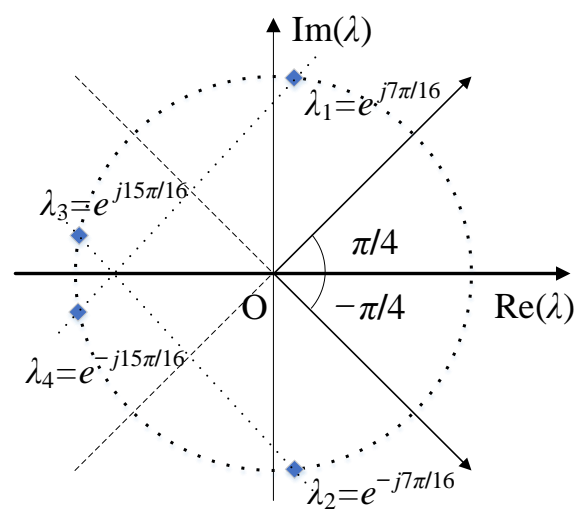

Fig. 4. Zero distribution of $P_{c}(\lambda)$ in Example 1

They are symmetric about $\{\lambda: \arg \{\lambda\}= \pm \pi / 4\}$. Also, it is seen that the $P(s)$ without pure imaginary zero can still be stable in the presence of the second singular case, which will never happen for polynomials with integer degrees.

Example 2: Consider the following fractional order linear $R L C$ circuit system (adopted from Example 4.4 [23]) in Fig. 5, where the capacitor and the coil have fractional order input-output property, 


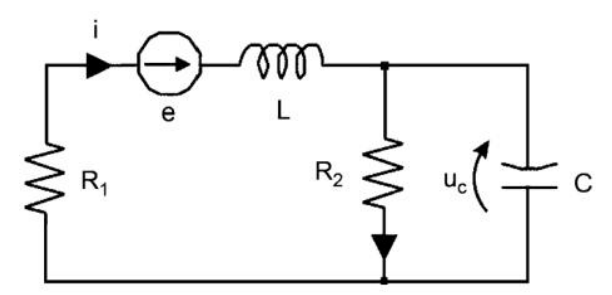

Fig. 5. A linear fractional RLC circuit system

$$
\left[\begin{array}{c}
\frac{d^{\alpha} U_{c}}{d t^{\alpha}} \\
\frac{d^{\beta} i}{d t^{\beta}}
\end{array}\right]=\left[\begin{array}{cc}
-\frac{1}{R_{2} C} & \frac{1}{C} \\
-\frac{1}{L} & -\frac{R_{1}}{L}
\end{array}\right]\left[\begin{array}{c}
U_{C} \\
i
\end{array}\right]+\left[\begin{array}{l}
0 \\
\frac{1}{L}
\end{array}\right] e .
$$

In order to make the system as positive, i.e., its trajectory starting from any nonnegative initial state remains forever in the positive orthant for all nonnegative inputs, a control law is designed in [23] as

$$
e=\left[\begin{array}{ll}
a+1 & b
\end{array}\right]\left[\begin{array}{c}
U_{c} \\
i
\end{array}\right], a>0, b>0
$$

Then the characteristic polynomial of the closed-loop system is

$$
P(s)=s^{\alpha+\beta}+\frac{R_{1}-b}{L} S^{\alpha}+\frac{1}{R_{2} C} S^{\beta}+\frac{R_{1}-a R_{2}-b}{R_{2} C L} .
$$

However, how to select the parameters $a$ and $b$ to stabilize the system was not further discussed. Here, we use the proposed Routh type test for this stabilization problem. As an illustration, suppose $\alpha=0.8$ and $\beta=0.4, R_{1}=1000 \Omega, R_{2}=2000 \Omega, L=0.05 \mathrm{H}, C=0.001 \mathrm{~F}$. Then we have the following Main Test table

$\begin{array}{cccc}-0.309 & 6180-6.18 b & 0.4045 & x_{1,4} \\ 0.9511 & 19022-19.02 b & 0.2939 & 0 \\ x_{3,1} & -0.5 & x_{3,3} & 0 \\ x_{4,1} & x_{4,2} & 0 & 0 \\ x_{5,1} & x_{5,2} & 0 & 0 \\ x_{6,1} & 0 & 0 & 0 \\ x_{7,1} & 0 & 0 & 0\end{array}$

where 


$$
\begin{aligned}
& x_{1,4}=10000-10 b-20000 a, \\
& x_{3,1}=12.36 b-12366, x_{3,3}=-x_{1,4} \\
& x_{4,1}=y_{1} / x_{3,1}, \quad y_{1}=235.114 b^{2}-470228.202 b+235114100, \\
& x_{4,2}=y_{2} / x_{3,1}, \quad y_{2}=19021.13 a+5.878 b-5877.853, \\
& x_{5,1}=y_{3} / y_{1}, \quad y_{3}=235114.10092 a b-380422.607 b-235114100.92 a \\
& \quad+190.211 b^{2}+190211303.02, \\
& x_{5,2}=x_{1,4}, \quad x_{6,1}=y_{4} / y_{3}, \\
& y_{4}= \\
& -361803398.88 a^{2}-89442719.1 a b^{3}+268328157300 a b^{2}- \\
& 268328157342679.8 a b+89442719142696677 a-44721.36 b^{4}+ \\
& 178885438.2 b^{3}-268328157209.5 b^{2}+178885438019081.4 b- \\
& 44721359459544933, \\
& x_{7,1}=-x_{1,4} \cdot
\end{aligned}
$$

Consider a nontrivial case, i.e., $x_{3,1} x_{5,1} x_{6,1} x_{7,1} \neq 0$ and $x_{1,4} x_{3,3} x_{4,2} x_{5,2} \neq 0$, then the stability condition, i.e., $n_{r}=0$, is analytically expressed as

$$
\begin{gathered}
n_{r}=\langle n \alpha / 2\rangle-\left(V_{f}-V_{l}\right)=\left[1+\operatorname{sgn}\left(x_{3,1}\right)+\operatorname{sgn}\left(y_{1}\right)+\operatorname{sgn}\left(x_{3,1} y_{3}\right)+\operatorname{sgn}\left(y_{1} y_{4}\right)-\right. \\
\left.\operatorname{sgn}\left(x_{1,4} y_{3} y_{4}\right)\right] / 2=0 .
\end{gathered}
$$

A gridding plot under this condition and the nontrivial condition $x_{3,1} \cdot x_{5,1} \cdot x_{6,1} \cdot x_{7,1} \cdot x_{1,4} \cdot x_{3,3}$. $x_{4,2} \cdot x_{5,2} \neq 0$ yields the stability region in the $a-b$ paratmeter space as shown in Fig. 6 . It implies that for $a>0$ and $b>0$, the stable region in $a-b$ paratmeter space is $1000-b-$ $2000 a>0$, i.e., $x_{1,4}>0$. This example shows an application of the proposed Routh-type test to a stabilization problem in addition to stability checking, which does outperform the methods from 
numerically solving zeros.

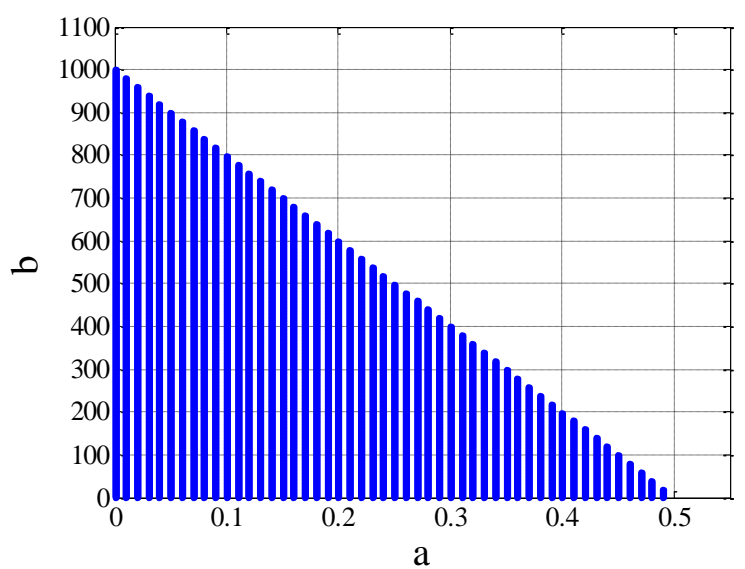

Fig. 6. Stability region in $a-b$ paratmeter space via gridding and scatter plot (colored points are within stability region)

Example 3: Consider a high order and ill-conditioned PCFD as

$$
P(s)=\left(s^{0.1}-2 s^{0.05}+4\right)^{36} .
$$

Its companion polynomial is $P_{c}(\lambda)=\left(\lambda^{2}-2 \lambda+4\right)^{36}$, which has 36 mutiple zeros at $1 \pm j \sqrt{3}$ with the arguments as $\pm \pi / 3$. Therefore, $P(s)$ is stable.

However, the zeros of $P_{c}(\lambda)$ solved by Matlab command "roots" show two positive real zeros $\lambda=1.5609$ and $\lambda=1.3662$. This implies that $P(s)$ is unstable, which is obviously incorrect.

On the other hand, the proposed Routh-type test for $P(s)$ yields $V_{f}=74, V_{l}=72, V_{f d}=$ $0, V_{d l}=0$ and $n_{i}(P)=0, n_{r}(P)=\langle 3.6 / 2\rangle-(74-72)=0$, i.e., it provides the correct result.

Here we present a quantitative comparison of computation costs among the above mentioned methods. They are $21.726 \mathrm{~ms}$ for our $P(s), 124.425 \mathrm{~ms}$ for the Matlab command "roots", and 95.938 ms for the LMI-based test, where the "roots" method gives a wrong result. Also, our Routh-type test is much faster than the LMI-based method about more than 4 times.

Example 4: Consider a polynomial with integer degrees

$$
P(s)=s^{8}+s^{7}+s^{6}+s^{5}+s^{2}+1
$$


It has the zero distribution triple as $(4,2,2)$. If classical Routh test is utilized, the first singular case occurs. We compare our test with available efficient methods, namely, Tsai and Chen's method [8], Pal and Kailath's method [11] and Genin's method [12], respectively as follows.

It can be verified that all these methods yield the correct result by their rules. Particularly, from our table (Table IV) we have $V_{f}=1, V_{l}=0, V_{d f}=0, V_{d l}=1, n_{i}=2, n_{r}=8 / 2-1-(1-$ $0)=2$, and $n_{l}=8-2-2=4$.

Therefore, it illustrates that our method with (20) and (25) is uniform for polynomials with integer and commensurate fractional degrees and treats the singular cases efficiently and simply.

Table I. Tsai and Chen's method

\begin{tabular}{|l|l|l|l|l|l|}
\hline$s^{8}$ & 1 & 1 & 0 & 1 & 1 \\
$s^{7}$ & 1 & 1 & & & \\
$\mathrm{M}_{0}$ & 0 & 0 & 1 & 0 & \\
$\mathrm{M}_{1}$ & 0 & 0 & 0 & 0 & \\
$\mathrm{M}_{2}$ & 0 & 0 & 0 & & \\
$s^{2}$ & 1 & 1 & & & \\
$s^{1}$ & 1 & & & & \\
$s^{0}$ & 2 & & & \\
\hline
\end{tabular}

Table II. Pal and Kailath's method

\begin{tabular}{|c|l|l|l|l|l|l|}
\hline$s^{8}$ & 1 & 1 & 0 & 1 & 1 & $\zeta$ \\
$s^{7}$ & 1 & 1 & & & & 1 \\
$s^{6}$ & 0 & 0 & 1 & 1 & & 0 \\
$s^{5}$ & 0 & 1 & 1 & & & 0 \\
$s^{4}$ & 0 & 1 & 1 & & & 0 \\
$s^{3}$ & 1 & 1 & & & & 1 \\
$s^{2}$ & 1 & 1 & & & & $1 / 2$ \\
$s^{1}$ & 2 & & & & & 2 \\
$s^{0}$ & 1 & & & & & \\
\hline
\end{tabular}

Table III. Genin's method

\begin{tabular}{|c|c|}
\hline$P_{0}(\mathrm{~s})=\mathrm{s}^{8}+s^{6}+s^{2}+1$ & $Q_{1}(\mathrm{~s})=s$ \\
\hline$P_{1}(\mathrm{~s})=s^{7}+s^{5}$ & $Q_{2}(\mathrm{~s})=s^{5}$ \\
\hline$P_{2}(\mathrm{~s})=s^{2}+1$ & $Q_{3}(\mathrm{~s})=1 / 2 s$ \\
\hline$P_{3}(\mathrm{~s})=2 s$ & $Q_{4}(\mathrm{~s})=2 s$ \\
\hline$P_{4}(\mathrm{~s})=1$ & \\
\hline
\end{tabular}


Table IV. Our LWW's method

\begin{tabular}{|c|c|c|c|c|}
\hline 1 & -1 & 0 & -1 & 1 \\
-1 & 1 & & & \\
21 & -1 & & & \\
1 & & & & \\
\hline
\end{tabular}

\section{CONCLUSION}

In this paper, Routh-type table tests of zero distribution have been presented for polynomials with commensurate fractional degrees (PCFDs) with respect to right half plane of Riemann principal sheet (the Main Test), the Riemann principal sheet (the Auxiliary Test), and general sector region (the General Test). Singular cases are treated and analyzed thoroughly, where the zero distribution feature phenomenon of polynomials with commensurate fractional degrees is revealed. It should be emphasized that Routh-type table test is the most efficient method with the least computation to obtain the zero distribution of PCFDs without actually solving zeros. This paper extends Routh tabular methods to PCFDs for theoretical completeness of Routh-type test on various polynomials and systems.

The main novelty of this paper includes the followings: (1) as the first in the literature to ask if there are Routh-type tests and methods for general commensurate fractional degree polynomials, (2) to present the uniform Routh-type tests and their uniform formulas to the analysis of zero distribution in the complex plane for both PCFDs and integer degree polynomials with either real or complex coefficients, (3) to keep the methods with some possible similarity to the classical Routh table test for its merit, (4) to handle the singular cases easily and correctly, (5) to reveal the underlying interesting symmetric property of zero distribution in the second singular case for the PCFDs, (6) to develop a new approach for analyzing the various systems stability and revealing the relationship between the polynomial data (commensurate order and coefficients) and their zero distributions for potential system design use, (7) to consider the Riemann Principal Sheet and the 
essential singularity point of the origin for the methods, and (8) the last but not least, to present the strict theoretical mathematical proof for the presenting Routh-type tests and methods.

Because of the importance of stability to various systems in different areas, the presented Routh-type tests will have significant impact to both theoretical and application areas in broad science and engineering fields. Noting that a series of results closely related to Routh test theory, such as the extended Hermite-Biehler theorem and Kharitonov theorem, applications to $H_{2} / H_{\infty}$ norm computation and model reduction, have made fruitful and successful applications in system analysis, we believe that the presented tests and theory will provide a new avenue for analysis and design of fractional order systems. We also expect similar extension of these applications to the fractional order systems based on the presented Routh-type table tests and theory.

\section{APPENDIX: TheORETICAl PROOFs For The MAin Results}

Proof of Theorem 1: The sketch of the proof is mainly along that of Gantmacher [2] for the proof of classical Routh table. Given a PCFD, the Argument principle is utilized, and the net phase change along the imaginary axis is associated with Cauchy index, which is computed by Sturm theorem and further leads to our derived Routh-type table. Detailed proof is as follows.

Consider a semicircle with a sufficient large radius $r$ and a center at the origin as in Fig. 7. It encircles all the right half plane principal zeros of $P(s)$ in (2) with real coefficients. Small semicircles with sufficient small radius $\varepsilon$ are utilized to exclude all pure imaginary principal zeros of $P(s)$ and the origin point (which is an essential singular point).

$$
\text { Let } \Gamma_{1}=\widehat{A B C D} \cup \cdots \cup \widehat{E F G} \cup \cdots \cup \widehat{H I J K} \text { from }-j \infty \text { to }+j \infty \text { and } \Gamma_{2}=\widehat{A Z K} \text { as }
$$
counterclockwise direction. The Argument principle implies that

$$
\lim _{r \rightarrow \infty} \Delta_{\Gamma_{2}+\left(-\Gamma_{1}\right)} \arg \{P(s)\}=2 n_{r} \pi
$$

where $\Delta_{\Gamma} \arg \{P(s)\}$ is the net phase change of $P(s)$ along any curve $\Gamma$. Meanwhile, since the 
highest degree term of $P(s)$ is $p_{n} s^{n \alpha}$, as the radius $r \rightarrow+\infty$, we have

$$
\lim _{r \rightarrow \infty} \Delta_{\Gamma_{2}} \arg \{P(s)\}=n \alpha \pi
$$

Then from (29) and (30), we have

$$
\frac{1}{\pi} \Delta_{\Gamma_{1}} \arg \{P(s)\}=n \alpha-2 n_{r}
$$

Let $s=j \omega, \omega \geq 0$, then it leads to

$$
\begin{gathered}
P(j \omega)=R(\omega)+j I(\omega), \\
\left\{\begin{array}{r}
R(\omega)=\sum_{k=0}^{n} p_{k} \cos (k \pi \alpha / 2) \omega^{k \alpha} \\
I(\omega)=\sum_{k=0}^{n} p_{k} \sin (k \pi \alpha / 2) \omega^{k \alpha}
\end{array}\right.
\end{gathered}
$$

Also, we have $\arg \{P(j \omega)\}=\arctan \frac{I(\omega)}{R(\omega)}$, for $\omega \geq 0$.

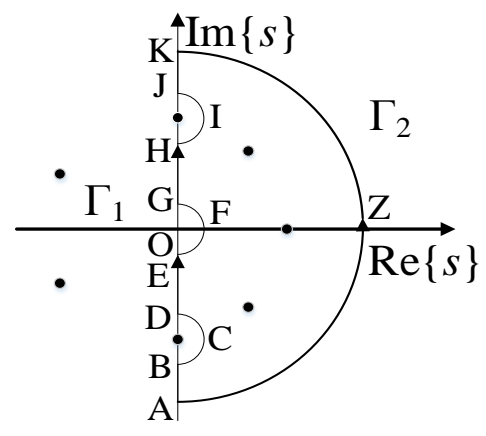

Fig. 7. Contour of the right half plane in $\mathcal{R P S}$

If $P(s)$ has no pure imaginary zero, then $\Gamma_{1}$ has only one semicircle centered at the origin, and $\lim _{\varepsilon \rightarrow 0} \Delta_{\Gamma_{1}} \arg \{P(s)\}=2 \Delta_{\omega=0}^{+\infty} \arctan \frac{I(\omega)}{R(\omega)}$. However, if $P(s)$ has $n_{i}$ pure imaginary zeros, we have

$$
\begin{gathered}
\lim _{\varepsilon \rightarrow 0} \Delta_{\Gamma_{1}} \arg \{P(s)\}-n_{i} \pi=2 \Delta_{\omega=0}^{+\infty} \arctan \frac{I(\omega)}{R(\omega)}, \\
n_{i}=2 d,
\end{gathered}
$$

where $d$ is the number of common positive real zeros of $R(\omega)$ and $I(\omega), \omega \in(0,+\infty)$, i.e., of the following $f_{g}(\omega)$ 


$$
f_{g}(\omega) \triangleq \operatorname{gcd}\{R(\omega), I(\omega)\}
$$

Next, we calculate the right hand sides of (34) by using Cauchy index:

$$
\begin{gathered}
\frac{1}{\pi} \Delta_{\omega=0}^{+\infty} \arctan \frac{I(\omega)}{R(\omega)}=-I_{0}^{+\infty} \frac{I(\omega)}{R(\omega)}+\tau, \\
\frac{1}{\pi} \Delta_{\omega=0}^{+\infty} \operatorname{arccot} \frac{R(\omega)}{I(\omega)}=I_{0}^{+\infty} \frac{R(\omega)}{I(\omega)}+v, \\
\tau=\frac{1}{\pi} \lim _{\omega \rightarrow+\infty} \arctan \frac{I(\omega)}{R(\omega)}-\frac{1}{\pi} \lim _{\omega \rightarrow 0} \arctan \frac{I(\omega)}{R(\omega)}, \\
v=\frac{1}{\pi} \lim _{\omega \rightarrow+\infty} \operatorname{arccot} \frac{R(\omega)}{I(\omega)}-\frac{1}{\pi} \lim _{\omega \rightarrow 0} \operatorname{arccot} \frac{R(\omega)}{I(\omega)} .
\end{gathered}
$$

For $n \alpha \in \mathbb{R}^{+} \backslash \mathbb{N}_{\text {odd }}$, i.e., $\rho=1$, we have $\operatorname{deg}(R(\omega)) \geq \operatorname{deg}(I(\omega))$. Then it follows from Sturm theorem that

$$
I_{0}^{+\infty} \frac{I(\omega)}{R(\omega)}=V(0)-V(+\infty)
$$

where $V(x)$ is the sign change at point $x$ of Sturm chain. Moreover, it is easy to observe that

$$
\begin{aligned}
& V(+\infty)=V\left(x_{1, n}, x_{2, n}, \cdots, x_{g, n}\right)=V_{f} . \\
& V(0)=V\left(x_{1, m_{1}}, x_{2, m_{2}}, \cdots x_{g, m_{g}}\right)=V_{l} .
\end{aligned}
$$

where $x_{1, n}, x_{2, n}, \cdots x_{g, n}$ and $x_{1, m_{1}}, x_{2, m_{2}}, \cdots x_{g, m_{g}}$ are the nonzero coefficients of the head highest terms and the tail lowest terms of $\left\{f_{1}(x), f_{2}(x), \cdots, f_{g}(x)\right\}$, respectively. Therefore, we have

$$
I_{0}^{+\infty} \frac{I(\omega)}{R(\omega)}=V(0)-V(+\infty)=V_{l}-V_{f} .
$$

Substituting (35), (37) and (44) into (34) and (31) yields

$$
n_{r}=n \alpha / 2-d-\left(V_{f}-V_{l}\right)-\tau, \text { for } \rho=1 .
$$

Calculating $\tau$ in (39), we have

$$
-0.5<\tau<0.5, \text { for } \rho=1,
$$




$$
n_{r}=\langle n \alpha / 2\rangle-d-\left(V_{f}-V_{l}\right), \quad \text { for } \quad \rho=1, n_{r} \in \mathbb{N},
$$

where $\langle\cdot\rangle$ is the round-off operator in (21).

Now we consider another case $n \alpha \in \mathbb{N}_{\text {odd }}$, i.e., $\rho=0$ and $\operatorname{deg}(R(\omega))<\operatorname{deg}(I(\omega))$. In a similar way to the previous case (but the Sturm chain here begins with $f_{1}(\omega)=I(\omega), f_{2}(\omega)=$ $R(\omega))$, we have

$$
\begin{aligned}
& v=\left\{\begin{aligned}
0.5, & \text { if } \sigma=+1 \\
-0.5, & \text { if } \sigma=-1
\end{aligned}\right. \\
& v=\sigma / 2=\operatorname{sgn}\left(x_{1, m_{1}} \cdot x_{2, m_{2}}\right) / 2 .
\end{aligned}
$$

where $v$ is in (38) and (40), and $\sigma$ is in (18). We have the following result similar to (45) as

$$
n_{r}=n \alpha / 2-d-\sigma / 2+\left(V_{f}-V_{l}\right), \text { for } \rho=0
$$

Finally, for either $\rho=1$ or $\rho=0$, combining (47) and (50) yields

$$
n_{r}=\left\langle n \alpha / 2-d-\sigma(1-\rho) / 2-(2 \rho-1)\left(V_{f}-V_{l}\right)\right\rangle \text {. }
$$

The first singular case occurs when the first few but not all elements of a row vanish in the Routh-type table. The shifting operation $\mathrm{O}$ is valid since it just accomplishes the Sturm theorem.

The second singular case occurs if there is an entire zero row. It implies $\operatorname{deg}\left(f_{g}(x)\right)>0$ where $f_{g}(x)$ is in (36). The function $f_{g}(x)$ and its companion polynomial $\tilde{f}_{g}(x)=f_{g c}(\lambda)$ have the same number of positive real zeros, i.e., the $d$ in (35).

Using Sturm theorem again, we calculate $d$ from the marked $\diamond$ row to the end row in the table by counting the sign changes. This completes the proof.

Proof of Theorem 2: The Auxiliary Test calculates the total number of principal zeros, i.e., in the $\mathcal{R P} \mathcal{S}$. The procedure of the Main Test for the RHP $\{s:-\pi / 2<\arg \{s\}<\pi / 2\}$ can also be applied to the $\mathcal{R P} \mathcal{S}$ as $\{s:-\pi<\arg \{s\} \leq \pi\}$. Denote $\tilde{d}$ and $\tilde{n}_{r}$ as the numbers of principal 
zeros on $\{s: \arg \{s\}=\pi\}$ and $\{s:-\pi<\arg \{s\}<\pi\}$, respectively. Then $\tilde{n}_{r}$ can be obtained similarly to (51) but to replace " $n \alpha / 2$ " and " $d$ " by " $n \alpha$ " and " $\tilde{d}$ " respectively as follows.

$$
\tilde{n}_{r}=\left\langle n \alpha-\tilde{d}-\sigma(1-\rho) / 2-(2 \rho-1)\left(V_{f}-V_{l}\right)\right\rangle .
$$

It is observed that $P(s)$ has $\tilde{d}$ principal zeros (with argument $+\pi$ ). And their conjugate ones are other $\tilde{d}$ adjoint zeros (with argument $-\pi$ ). Then the total number of principal zeros is $n_{p}=\tilde{n}_{r}+\tilde{d} \quad$ which $\quad$ is exactly the same as

Proof of Theorem 3: Consider $P(s)$ with $P(j \omega)=R(\omega)+j I(\omega), \omega \geq 0$ and the equation of companion polynomials as $P_{c}\left((j \omega)^{\alpha}\right)=R_{c}\left(\omega^{\alpha}\right)+j I_{c}\left(\omega^{\alpha}\right)$, for $\omega \geq 0$. By setting $\omega^{\alpha}=x$, we have $P_{c}\left(e^{j \pi \alpha / 2} x\right)=R_{c}(x)+j I_{c}(x)$, for $x \in \mathbb{R}^{+}$. By analytic continuation from $\mathbb{R}^{+}$to $\mathbb{C}$, we have $P_{c}\left(e^{j \pi \alpha / 2} x\right)=R_{c}(x)+j I_{c}(x)$, for $x \in \mathbb{C}$. Set $x=\gamma^{\alpha}$, then there holds $P(j \gamma)=R(\gamma)+j I(\gamma)$, for $\gamma \in \mathbb{C}$.

The second singular case occurs if and only if $\operatorname{deg}\left(f_{g}(x)\right)>0$ where $f_{g}(x)$ is in (36). From above, there holds

$$
f_{g}(\gamma)=0 \Leftrightarrow R(\gamma)=I(\gamma)=0 \Leftrightarrow P(j \gamma)=0
$$

Since $f_{g}(\cdot)$ and $P(\cdot)$ are real coefficient polynomials, we have

$$
f_{g}(\gamma)=0 \Leftrightarrow P(s)=0, s \in\left\{j \gamma,-j \gamma^{*}, j \gamma^{*},-j \gamma\right\}
$$

This completes the proof.

Proof of Theorem 4: Consider $\Gamma_{1}$ and $\Gamma_{2}$ as shown in Fig. 2, it follows from the Argument principle that

$$
2 n_{\Omega} \pi=\left(\theta_{2}-\theta_{1}\right) n \alpha+\Delta_{\Gamma_{1}-\Gamma_{2}} \arg \{P(s)\}
$$

In the same way as the procedure of the Main Test, we have 


$$
\begin{aligned}
& \frac{1}{\pi} \Delta_{\Gamma_{1}} \arg \{P(s)\}=\left(2 \rho_{1}-1\right) \Phi_{1}+\tau_{1} \rho_{1}+\sigma_{1}\left(1-\rho_{1}\right) / 2-n_{\partial \Omega}^{(1)} \\
& \frac{1}{\pi} \Delta_{\Gamma_{2}} \arg \{P(s)\}=\left(2 \rho_{2}-1\right) \Phi_{2}+\tau_{2} \rho_{2}+\sigma_{2}\left(1-\rho_{2}\right) / 2+n_{\partial \Omega}^{(2)}
\end{aligned}
$$

where $\Phi_{1}, \Phi_{2}, \rho_{1}, \rho_{2}, \sigma_{1}$, and $\sigma_{2}$ are determined by the tables corresponding to $\theta_{1}$ and $\theta_{2}$,

respectively. Also, we have $-0.5<\tau_{1}, \tau_{2}<0.5$. The term $n_{\partial \Omega}^{(1)}$ in (56) and the term $n_{\partial \Omega}^{(2)}$ in (57) are with different signs because the small semicircles of $\Gamma_{1}$ and $\Gamma_{2}$ as in Fig. 2 are with different directions.

Let $\Phi_{0} \triangleq \frac{\left(\theta_{2}-\theta_{1}\right) n \alpha}{2 \pi}-\frac{k_{\partial \Omega}^{(1)}+k_{\partial \Omega}^{(2)}}{2}-\frac{\sigma_{2}\left(1-\rho_{2}\right)-\sigma_{1}\left(1-\rho_{1}\right)}{4}$. Substituting (56) and (57) into (55) and noting that $n_{\Omega}$ must be an integer, we have the following uniform expression:

$$
n_{\Omega}=\left\langle\Phi_{0}+\left(\rho_{1}-0.5\right) \Phi_{1}-\left(\rho_{2}-0.5\right) \Phi_{2}\right\rangle
$$

The remaining is to prove $n_{\partial \Omega}^{(1)}=d_{1}$ and $n_{\partial \Omega}^{(2)}=d_{2}$ corresponding to $\theta_{1}$ and $\theta_{2}$ respectively. This is similar to that in the Main Test proof and is omitted here.

\section{REFERENCES}

[1] E. J. Routh, A Treatise on the Stability of a Given State of Motion. London, U.K.: Macmillan, 1877.

[2] F. R. Gantmacher, The Theory of Matrices vol. II. New York: Chelsea, 1959.

[3] M. T. Ho, A. Datta, and S. P. Bhattacharyya, "An elementary derivation of the Routh-Hurwitz criterion," IEEE Transactions on Automatic Control, vol. 43, pp. 405-409, 1998.

[4] A. Ferrante, A. Lepschy, and U. Viaro, "A simple proof of the Routh test," IEEE Transactions on Automatic Control, vol. 44, pp. 1306-1309, 1999.

[5] H. Lev-Ari, Y. BistritZ, and T. Kailath, "Generalized Bezoutians and families of efficient zero-location procedures," IEEE Transactions on Circuits and Systems, vol. 38, no. 2, pp. 170-186, 1991.

[6] S. S. Chen and J. S. H. Tsai, "A new tabular form for determining root distribution of a complex polynomial with respect to the imaginary axis," IEEE Transactions on Automatic Control, vol. 38, pp. 1536-1541, 1993.

[7] K. P. Sondergeld, "A generalization of the Routh-Hurwitz stability-criteria and an application to a problem in robust controller-design," IEEE Transactions on Automatic Control, vol. 28, pp. 965-970, 1983. 
[8] J. S. H. Tsai and S. S. Chen, "Root distribution of a polynomial in subregions of the complex-plane," IEEE Transactions on Automatic Control, vol. 38, pp. 173-178, 1993.

[9] Y. Bistritz, "Optimal fraction-free Routh tests for complex and real integer polynomials," IEEE Transactions on Circuits and Systems I-Regular Papers, vol. 60, pp. 2453-2464, 2013.

[10] S. K. Pillai, "The epsilon-method of the Routh-Hurwitz criterion," IEEE Transactions on Automatic Control, vol. 26, pp. 584-584, 1981.

[11]D. Pal and T. Kailath, "Displacement structure approach to singular root distribution problems: The imaginary axis case," IEEE Transactions on Circuits and Systems I: Fundamental Theory and Applications, vol. 41, no. 2, pp. 138-148, 1994.

[12] Y. V. Genin, "Euclid algorithm, orthogonal polynomials, and generalized Routh-Hurwitz algorithm," Linear Algebra and its Applications, vol. 246, pp. 131-158, 1996.

[13] M. A. Choghadi and H. A. Talebi, "The Routh-Hurwitz stability criterion, revisited: the case of multiple poles on imaginary axis," IEEE Transactions on Automatic Control, vol. 58, pp. 1866-1868, 2013.

[14] L. Qiu, "The renaissance of Routh," in SICE 2004 Annual Conference, vol. 2. IEEE, 2004, Conference Proceedings, pp. 1220-1222.

[15]C. Yan, S.-G. Wang and X. Zeng, "A new method for multi-parameter robust stability distribution analysis of linear analog circuits," Proc. IEEE/ACM International Conference on Computer-Aided Design (ICCAD) 2011, pp.420-427, San Jose, CA, USA, Nov. 2011.

[16] N. S. Nise, Control Systems Engineering, 7th Edition. Wiley, Chapter 6, pp.299-334, 2014.

[17]I. Podlubny, "Fractional-order systems and $P I^{\lambda} D^{\mu}$-controllers," IEEE Transactions on Automatic Control, vol. 44, pp. 208-214, 1999.

[18]C. A. Monje, Y. Q. Chen, B. M. Vinagre, D. Xue, and V. Feliu, Fractional-order Systems and Controls:Fundamentals and Applications. London: Springer-Verlag, 2010.

[19]A. S. Elwakil, "Fractional-order circuits and systems: an emerging interdisciplinary research area," IEEE Circuits and Systems Magazine, vol. 10, pp. 40-50, 2010.

[20] M. O. Efe, "Fractional order systems in industrial automation-A survey," IEEE Transactions on Industrial Informatics, vol. 7, pp. 582-591, 2011.

[21] S. Westerlund and L. Ekstam, "Capacitor theory," IEEE Transactions on Dielectrics and Electrical Insulation, vol. 1, no. 5, pp. 826-839, 1994.

[22]I. Schäfer and K. Krüger, "Modelling of lossy coils using fractional derivatives," Journal of Physics D: Applied Physics, vol. 41, no. 4, p.045001, 2008.

[23] T. Kaczorek, "Positive linear systems consisting of $n$ subsystems with different fractional orders," IEEE Transactions on Circuits and Systems I-Regular Papers, vol. 58, pp. 1203-1210, 2011.

[24]R. K. H. Galvao, S. Hadjiloucas, K. H. Kienitz, H. M. Paiva, and R. J. M. Afonso, "Fractional Order Modeling of Large Three-Dimensional RC Networks," IEEE Transactions on Circuits and Systems I-Regular Papers, vol. 60, pp. 624-637, 2013.

[25] J. Wang, and Y. Zhang, "On the concept an existence of solutions for fractional impulsive systems with Hadamard derivatives," Applied Mathematics Letters, vol. 35, pp. 85-90, 2015.

[26]J. Wang, A. G. Ibrahim, and M. Feckan, "Nonlocal impulsive fractional differnetial inclusions with fractional sectorial operaters on Banach spacs," Applied Mathmatics and 
Computation, vol. 257, pp. 103-118, 2015.

[27]D. Matignon, "Stability properties for generalized fractional differential systems," ESAIM: Proc., vol. 5, pp. 145-158, 1998.

[28] R. Malti, X. Moreau, F. Khemane, and A. Oustaloup, "Stability and resonance conditions of elementary fractional transfer functions," Automatica, vol. 47, pp. 2462-2467, 2011.

[29] J. Sabatier, M. Moze, and C. Farges, "LMI stability conditions for fractional order systems," Computers \& Mathematics with Applications, vol. 59, pp. 1594-1609, 2010.

[30]C. Farges, M. Moze, and J. Sabatier, "Pseudo-state feedback stabilization of commensurate fractional order systems," Automatica, vol. 46, pp. 1730-1734, 2010.

[31]F. Merrikh-Bayat, M. Afshar, and M. Karimi-Ghartemani, "Extension of the root-locus method to a certain class of fractional-order systems," ISA Transactions, vol. 48, no. 1, pp. 48-53, 2009.

[32]I. Petráš, "Stability of fractional-order systems with rational orders: a survey," Fractional Calculus \& Applied Analysis, vol. 12, pp. 269-298, 2009.

[33] M. Rivero, S. V. Rogosin, J. A. Tenreiro Machado, and J. J. Trujillo, "Stability of fractional order systems," Mathematical Problems in Engineering, vol. 2013, 2013.

[34]I. Koca, "Mathematical modeling of nuclear family and stability analysis," Applied Mathematical Sciences, vol. 8, no. 68, pp. 3385-3392, 2014.

[35]E. Ahmed, A. M. A. El-Sayed, and H. A. A. El-Saka, "On some Routh-Hurwitz conditions for fractional order differential equations and their applications in Lorenz, Rossler, Chua and Chen systems," Physics Letters A, vol. 358, no. 1, pp. 1-4, 2006.

[36]J. Sabatier, C. Farges, and J. C. Trigeassou, "A stability test for non-commensurate fractional order systems," Systems \& Control Letters, vol. 62, pp. 739-746, 2013.

[37] V. Y. Pan, "Solving a polynomial equation: some history and recent progress," SIAM Review, vol. 39, pp. 187-220, 1997.

[38]A. Nemirovskii and P. Gahinet, "The projective method for solving linear matrix inequalities," in American Control Conference, vol. 1. IEEE, pp. 840-844, 1994.

[39] S. Sherman, "LIV. Generalized Routh-Hurwitz discriminant (An Extension of the Theorems of Sturm, Routh, and Huruitz, on the Roots of Polynomial Equations.)," Philosophical Magazine, vol. 37, pp. 537-551, 1946.

[40]M. Marden, Geometry of polynomials. American Mathematical Soc., 1966. 